\title{
THE TOPOGRAPHY OF CYTOGENESIS IN THE DEVELOPING RETINA OF THE CAT ${ }^{1}$
}

\author{
DAVID H. RAPAPORT ${ }^{2}$ AND JONATHAN STONE \\ School of Anatomy, University of New South Wales, Sydney, Australia \\ Received October 26, 1982; Revised February 25, 1983; Accepted April 7, 1983
}

\begin{abstract}
We have studied the pattern of cytogenesis in the developing retina of the cat, by observing mitotic cells in sections and whole mounts of the retinae of animals between the ages of $\mathrm{E}$ (cmbryonic day) 29 and $P$ (postnatal day) 20. The whole mounts were prepared with the mitotic or ventricular layer uppermost; all of the mitotic cells in this layer could then be surveyed. In retinae from animals up to E46, mitotic cells were present in the ventricular layer at densities of 1000 to $3000 \mathrm{cells} / \mathrm{mm}^{2}$, and their density did not vary consistently with position in the retina. Thus cell division occurs throughout the retina at these ages, with an approximately constant spatial density. By E50, cytogenesis begins to cease and there is a significant pattern to the cessation. Initially, mitotic activity ceases over a small region of retina at the site of the developing area centralis. The nonmitotic area then increases with age, comprising the central 30 to $50 \%$ of the retina at birth and the whole of the retina by $\mathrm{P} 10$. The pattern of cessation of cytogenesis is closely coincident in space and time with the development of the outer plexiform layer and the maturation of the ganglion cell layer described in previous studies. These patterns presumably contribute to the regional variations in structure apparent in the adult retina.
\end{abstract}

In the developing retina, cells undergoing mitosis are found in a narrow layer at its outer surface, next to the external limiting membrane. Early in the development of the eye this surface of the retina is separated from the pigment epithelium by the optic vesicle, the extension of the forebrain vesicle into the optic stalk. The layer of mitotic cells which forms at this surface during cytogenesis of the retina thus corresponds to the "ventricular layer" of the rest of the developing neural tube (Angevine et al., 1970). It does not persist in the mature retina, being replaced by the cell bodies of receptors.

Cells leaving the mitotic cycle are thought to migrate radially inward from the ventricular layer to their eventual position in one of the inner layers of the retina (Sidman, 1961; Morest, 1970). Recently, Johns (1982) reported evidence that in the goldfish retina the genesis of some receptor cells occurs some distance from the

\footnotetext{
${ }^{1}$ This work was supported by the National Health and Medical Research Council of Australia, by National Institutes of Health Grant EY02671 to J. S., and by National Institutes of Health Postdoctoral Fellowship EY 05433 to D. H. R. We wish to thank Mss. Y. Cheska, J. Duncan, K. Hickson, and M. Wegner for technical assistance and B. Dreher for helpful comments on the manuscript.

${ }^{2}$ To whom correspondence should be addressed, at School of Anatomy, University of New South Wales, P. O. Box 1, Kensington, New South Wales 2033, Australia.
}

ventricular surface. The cells generated at this site migrate outward to the receptor layer. Similar evidence has not yet emerged in studies of the mammalian retina; mitotic cells have been observed only in the ventricular layer, and the direction of cell migration is assumed to be inward.

If a developing retina is dissected away from the sclera, choroid, and pigment epithelium and prepared as a whole mount with its ventricular surface uppermost, the full population of mitotic cells in the ventricular layer at the time of death can readily be observed. Because the cells are intact, and because the axes of the mitotic spindles are (as elsewhere (Martin, 1967)) oriented predominantly parallel to the ventricular surface, it is relatively easy to interpret the stage of the mitotic cycle reached by individual cells. Because the retina is intact, it is relatively easy to reconstruct the topography of mitotic cells.

This paper describes patterns in the distribution of mitotic cells over the ventricular surface of the cat's retina during fetal and early postnatal life, and discusses the significance of the patterns in the development of the topography of the adult retina.

\section{Materials and Methods}

Material. Eyes were obtained from kittens of known postnatal age and from fetuses removed from pregnant animals by Caesarian section under general anesthesia 
and strict asepsis. The gestational age of the fetuses was determined by one of two methods. The first method (Stone et al., 1982) involved exposing an estrous female to a potent male for $24 \mathrm{hr}$, that period being taken as embryonic day 1 (E1) of a resulting pregnancy. In the second method ( $\mathrm{Ng}$ and Stone, 1982; Rapaport and Stone 1982), the gestational age of the fetus studied was estimated from the date of birth of littermates brought to term and delivered normally, assuming gestation to have lasted 65 days. Using either method, the accuracy of timing was less than $24 \mathrm{hr}$. However, when the material used was considered in the chronological order established by these methods, a pattern of steady development emerged, suggesting that the accuracy of timing was adequate for the study.

The eyes were fixed by either immersion in or perfusion with $10 \%$ formalin, or by perfusion with a mixture of $1 \%$ glutaraldehyde and $2.5 \%$ paraformaldehyde in 0.1 M sodium cacodylate buffer ( $\mathrm{pH}$ 7.4), followed by immersion in $10 \%$ formalin.

Preparation of whole mounts. Whole mount preparations of the retina were prepared by conventional dissection and staining (Stone, 1981). The retina was dissected free from the other layers of the eyeball, spread on a (nongelatinized) glass slide with the ganglion cell layer up, and carefully cleaned of vitreous humor. The retina was then floated off the slide into saline and mounted on a gelatinized slide, with its ventricular (in older animals its receptor) layer uppermost and carefully blotted dry. The slide carrying the retina was then placed in formalin vapor at room temperature and left overnight, so that the retina became tightly adherent to the slide. The retina was stained with cresyl violet. The staining protocol was that used for staining the ganglion cell layer (Stone, 1981), but shorter staining times (5 min) seemed optimal. Retinae were analyzed for nine prenatal animals (E24, E29, E41, E42, E46 (three animals), E50, E57) and four postnatal animals (P1 (2 animals), P5, P10).

This procedure was fairly reliable, the principal problems being the small size and fragility of fetal retinae, and the adherence of the pigment epithelium and of pigment from the choroid layer to the external limiting membrane. When just the pigment epithelium remained adherent it was often possible to stain and observe cells in the ventricular layer satisfactorily. Adherent choroidal pigment usually obscured the ventricular layer, however. The adherence of pigment was unpredictable but seemed less a problem with formalin fixation; for this reason retinae fixed with glutaraldehyde/paraformaldehyde were postfixed in formalin (see above).

Mapping of mitotic activity in whole mounts. The identification of mitotic figures is described in the first section under "Results." Their distribution over the retina was mapped under the microscope using conventional protocols (Stone, 1981). The density of mitotic cells was measured with a calibrated eyepiece graticule, using a $x$ 100 objective. Counts were made at $1-$ or $0.5-\mathrm{mm}$ intervals across the full extent of each retina.

Preparation of retinal sections. These sections were prepared for an earlier study (Rapaport and Stone, 1982). Eyes were obtained and fixed as described above. They were then dehydrated, cleared, and embedded in paraffin.
Sections were cut with a rotary microtome at $8 \mu \mathrm{m}$ and were stained with hematoxylin and eosin. Eyes from animals of five ages (E32, E37, E51, E57, and P1) were examined in sections.

Estimating the orientation of mitotic spindles. Cells in anaphase and telophase were identified in sections of the retina by the parallel orientation of two linear aggregations of chromosomal material (Fig. 1 and 2). Since the mitotic spindle itself was not apparent, we assumed the axis of the spindle to be at right angles to the long axis of the chromosome aggregations. For each retina, samples of 30 to 40 cells in anaphase or telophase were drawn with a camera lucida; for each cell the segment of the external limiting membrane adjacent to the cell was also drawn. The angle between the mitotic spindle and the retinal surface was measured graphically (Fig. 2, top).

\section{Results}

\section{Appearance and interpretation of mitotic cells}

Appearance. Mitotic cells were recognized in whole mount preparations by the intense staining of their chromosomal material with cresyl violet and by the characteristic shapes formed by that material. In the sections (Fig. 1, bottom), which were stained with hematoxylin and eosin, mitotic cells stained only slightly more darkly than did neighboring cells, and were recognized by the shapes formed by their chromosomal material. In both whole mount preparations and sections, mitotic cells appear to be restricted to a "layer" immediately adjacent to the external limiting membrane of the retina. The separation of mitotic cells from the external limiting membrane did not exceed $10 \mu$, and nonmitotic cells were not observed between mitotic cells and the membrane.

Stages of mitosis. The identification of the stage of mitosis reached by a particular cell was considerably easier in whole mounts, in which all cells are intact, than in sections. Prophase is characterized by the separation of the centrioles to different poles of the cell, the formation of a microtubular spindle between them, the dissolution of the nuclear membrane, and the disappearance of the nucleolus (where present). The chromosomes then begin to intertwine with the spindle. Because cells of the neuroblast layer of the retina lack nucleoli even during interphase, and because we could not reliably observe either the nuclear membrane or the mitotic spindle, cells in prophase ( $p$ in Fig. 1) were identified by the lack of orientation in their chromosomal material.

Cells in metaphase ( $m$ in Fig. 1) were identified by the single linear array formed by their chromosomes, as they arrange themselves along the equator of the mitotic spindle. In cells in ana- and telophase ( $a-t$ in Fig. 1), the sets of chromosomes destined for the two daughter cells separate along the spindle, forming two linear chromosomal figures which lie parallel to each other. The feature which distinguishes telophase from anaphase is the formation of a cleavage furrow around the equator of the cell, between the two sets of chromosomes; the furrow subsequently deepens until the daughter cells are fully separate. The cleavage furrow could not be identified reliably in our preparations, and we have not, therefore, distinguished these two phases. For counting purposes, 

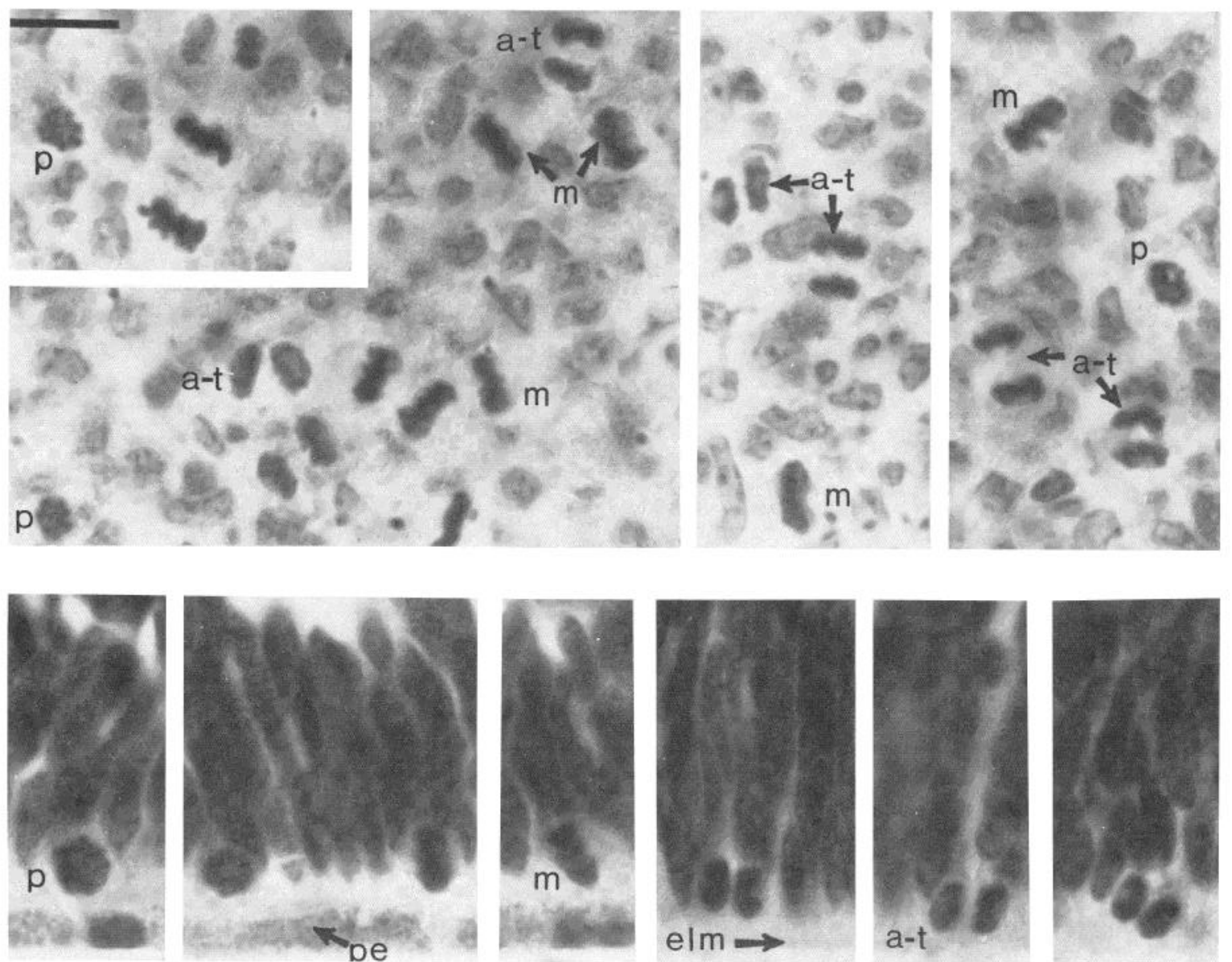

Figure 1. Top, Regions of the ventricular layer from a whole mount of an E50 retina stained with cresyl violet, showing cells at various stages of mitosis: prophase $(p)$, metaphase $(m)$, and ana- or telophase $(a-t)$. Bottom, Paraffin sections $(8 \mu \mathrm{m})$ of retina from an E57 cat fetus stained with hematoxylin and eosin. Note mitotic cells in prophase $(p)$, metaphase $(m)$ and ana- or telophase $(a-t)$. The pigment epithelium is labeled pe, and the external limiting membrane is labeled elm. Note the obliquity of the chromosomal figures in the two panels at the right. The scale bar represents $10 \mu$.

each pair of chromosomal figures was recorded as a single mitotic cell.

A small minority of mitotic cells could not be readily identified as being in one of the stages just discussed, usually because they appeared to be transitional between two stages. They did not seem sufficiently numerous to prejudice the findings presented below.

\section{The orientation of mitotic spindles}

Two issues are involved in the orientation of mitotic spindles. The first is technical. If a spindle were perpendicular to the external limiting membrane, one chromosome group of an anaphase or a telophase cell would, in a whole mount preparation, tend to obscure the other, and the cell might be mistakenly interpreted as in metaphase. The second issue is related (Martin, 1967) to Sauer's (1935) model of the interkinetic migration of nuclei in the developing neural tube, and is considered further under "Discussion."

Obliquely oriented mitotic cells were not easily recognized in whole mount preparations, but they were readily seen in sections (Fig. 1, bottom). To estimate the fraction of mitotic cells in which the spindle is obliquely oriented, we surveyed a series of sections from each of five retinae (E32, E37, E51, E57, and P1), and for each cell encountered in anaphase and telophase we estimated the angle between the axis of its mitotic spindle and the external limiting membrane (as in Fig. 2, top). Figure 2 shows 10 ana- or telophase figures observed consecutively in an E32 retina, and Figure 3 shows frequency/obliquity histograms from four of the retinae studied; the results obtained from the remaining retina were fully consistent. In all four retinae, the mean obliquity of the spindle was less than $15^{\circ}$, and in the great majority of the cells encountered (34 of 37,33 of 36,37 of 37 , and 29 of 29 , respectively) obliquity was $30^{\circ}$ or less. Presumably, the obliquity of spindle axes causes only a small error in the identification in whole mounts of cells in anaphase or telophase.

\section{Distribution of mitotic cells}

Figure 4 shows a map of the distribution of mitotic cells in the retina of an E50 cat fetus. The numbers in Figure 4, top, indicate the density of cells in mitosis (all stages), while the more schematic diagram in Figure 4, bottom, summarizes regional variations in the distribu- 

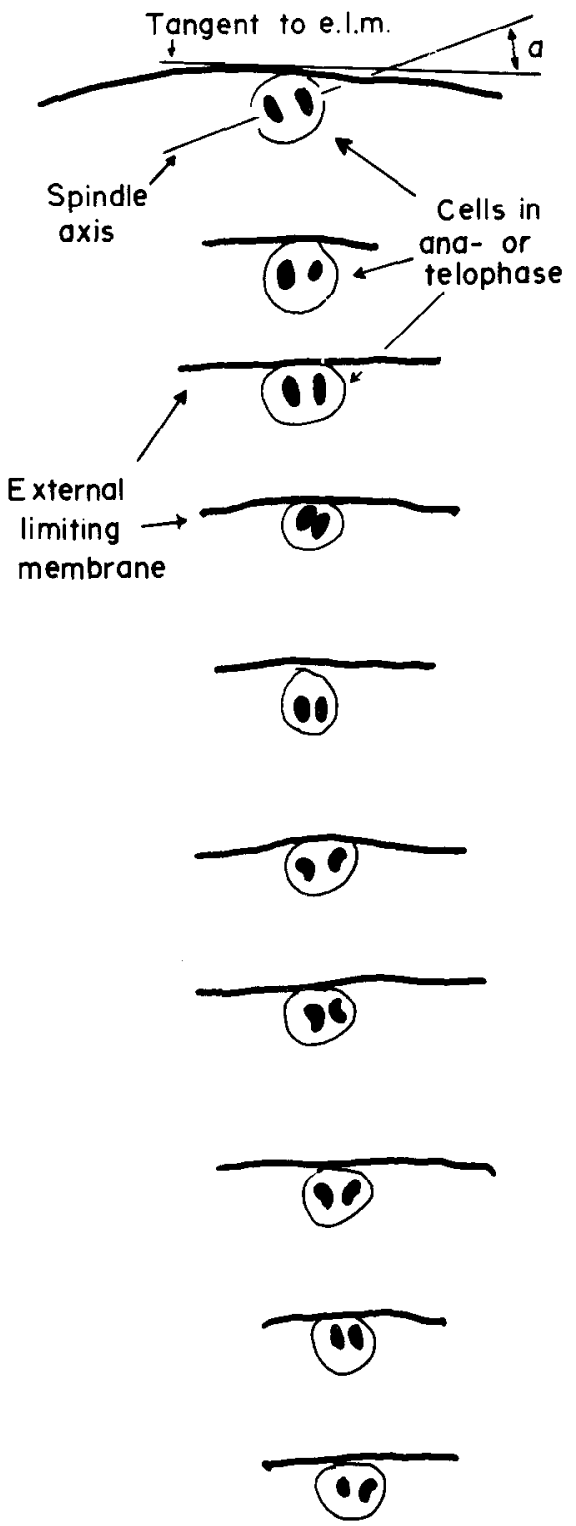

Figure 2. Camera lucida drawings of 10 cells in ana- or telophase, encountered consecutively in sections of an E32 retina. For each, the two chromosome sets of the dividing cell are drawn in black, and the external limiting membrane is also shown. The cell membrane is also drawn, although in some cells it was only partially visible. In the drawing at the top we indicate the way in which the obliquity of a mitotic spindle was estimated. A line was drawn through the chromosome sets, as nearly as possible at right angles to their long axes; this was taken to be the axis of their mitotic spindle. A second line was drawn tangential to the external limiting membrane at the position of the cell. The angle $a$ between these two lines was recorded as the obliquity of the axis of the cell's mitotic spindle.

tion of mitotic cells. Four features of the map deserve comment.

1. Over most of the retina the density of mitotic cells falls in the range of 11 to 33 cells $/ 0.01 \mathrm{~mm}^{2}$, with little apparent pattern to the variations in density.

2. A region of zero or very low $\left(<500\right.$ cells $\left./ \mathrm{mm}^{2}\right)$ mitotic activity (a "cold spot") is apparent several disk diameters (2 to $3 \mathrm{~mm}$ ) temporal to the optic disk; i.e., in the region in which the area centralis develops. The appearance of the ventricular layer in this region is shown in Figure $5 \mathrm{~A}$.

3. Surrounding the cold spot is a "cool" region where the density of mitotic cells was somewhat higher (500 to 1000 cells $/ \mathrm{mm}^{2}$ ), but still below levels in peripheral retina.

4. The regions of zero and low mitotic activity are not circular but horizontally elongated. For the area bounded by the 500 cells $/ \mathrm{mm}^{2}$ line, for instance, the ratio of its horizontal to its vertical extent is $1.8: 1$; for the 1000 cells $/ \mathrm{mm}^{2}$ line the corresponding ratio is $1.4: 1$.

Figure 6 shows maps of the distribution of mitotic cells in the ventricular layer of three retinae younger than E50; namely, E29, E41, and E46. The retina grows very considerably in this period, from $7.5 \mathrm{~mm}^{2}$ to $55 \mathrm{~mm}^{2}$, but the following features seem common to all three retinae.

1. The density of mitotic cells ranged between 1000 and 2500 cells $/ \mathrm{mm}^{2}$ and did not change consistently with age.

2. There was no apparent pattern to the distribution
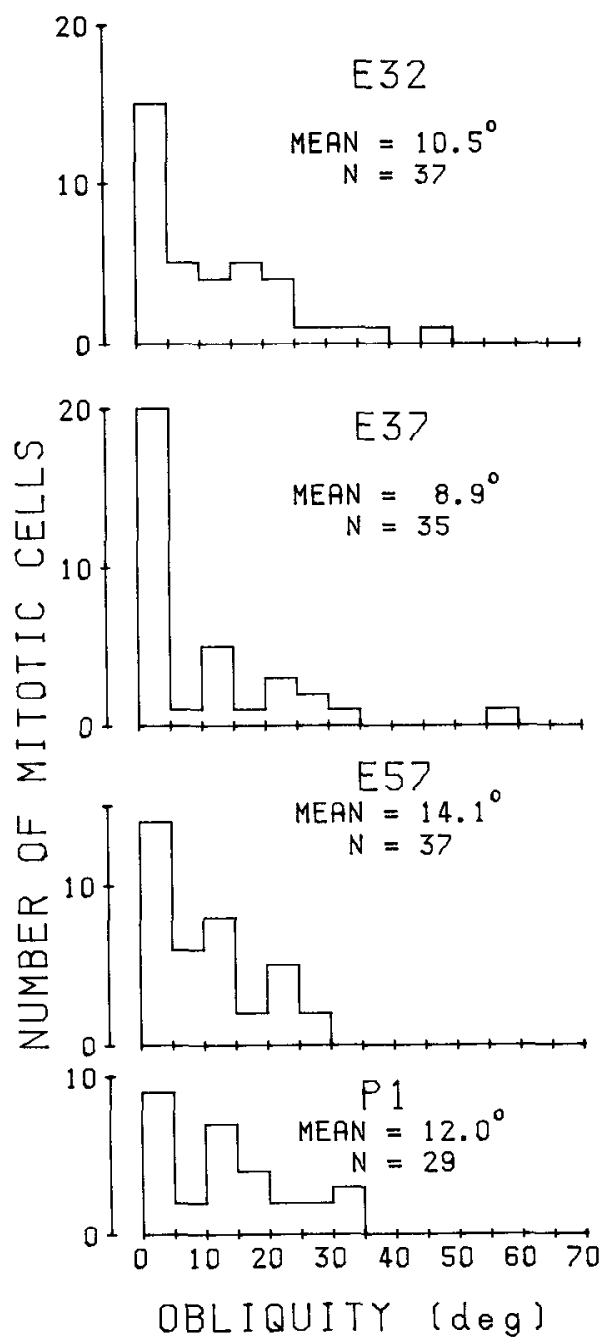

Figure 3. Frequency/obliquity histograms for cells in anaphase or telophase, in the retinae of fetuses aged E32, E37, and E57, and in the retina of a neonatal (P1) kitten. Note that in the great majority of cases the mitotic spindle is parallel to the ventricular surface, or no more than $30^{\circ}$ oblique to it. 

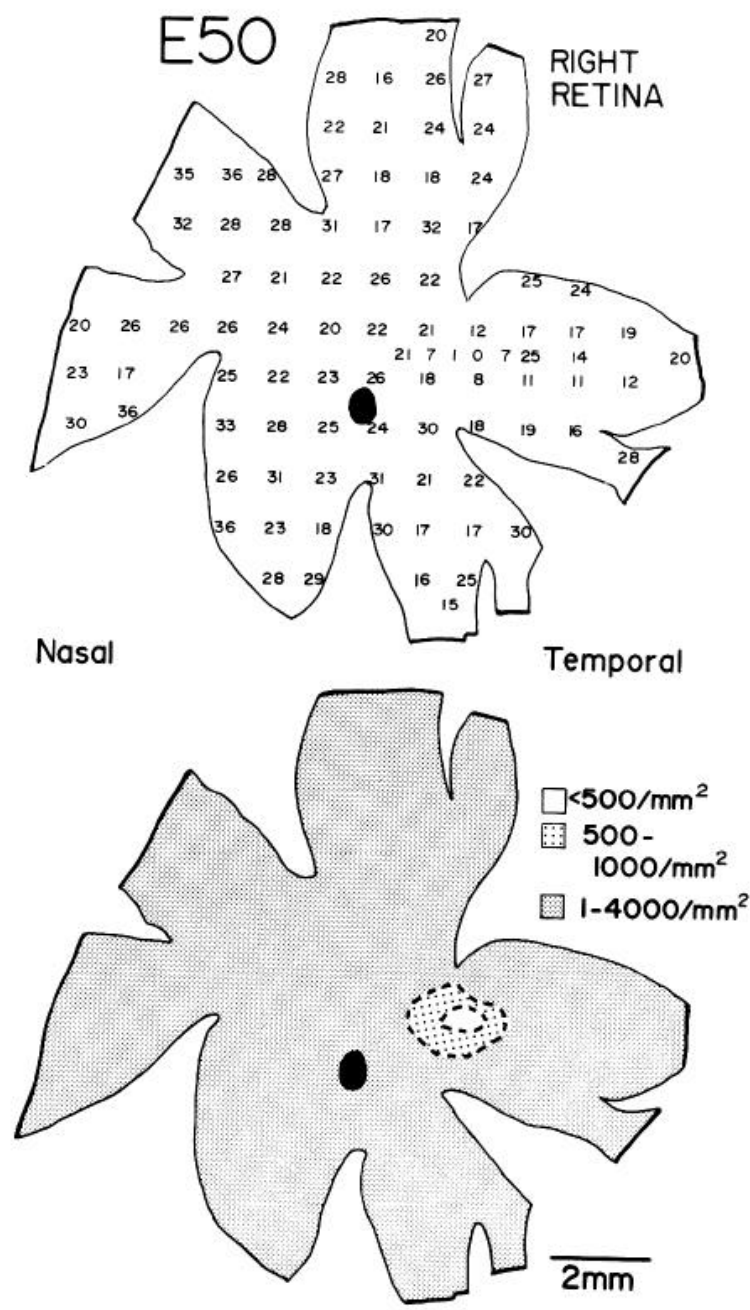

Figure 4. Maps of the distribution of mitotic cells in the ventricular layer of the retina of an E50 cat fetus. The two maps are of the same right retina. The map at the top shows part of the matrix of counts used to construct the more schematic map at the bottom. Each count is the number of mitotic cells in an area of $0.01 \mathrm{~mm}^{2}$. The counts vary considerably, but the only consistent variation was in a region a little above and temporal to the optic disc, where a group of low counts is apparent. The bottom map shows the small region of near-zero mitotic activity, and the surrounding "anulus" of low counts of mitotic cells (coarse dots). Over most of the retina (fine dots) the density of mitotic cells varies between 1100 and 3300 cells/ $\mathrm{mm}^{2}$.

of mitotic cells; they appeared to be approximately uniformly distributed.

3 . The mitotic cells did not vary noticeably with age in size or conformation (Figure 7).

Figure 8 shows maps of the distribution of mitotic cells in the ventricular layer of three retinae older than E50; namely, E57, P1, and P5. The zone of zero to low mitotic activity expands during this period, covering $0.5 \%$ of the retina at $\mathrm{E} 51,1.8 \%$ at $\mathrm{E} 57,30$ to $52 \%$ at $\mathrm{P} 1$ (two retinae mapped), and $42 \%$ at P5. High spatial densities (2000 to 4000 cells $/ \mathrm{mm}^{2}$ ) of mitotic cells seemed to be confined to the periphery of the E57 retina and were not found at P1 or later ages.

In a $\mathrm{P} 10$ retina, no mitotic cells were detected, even at
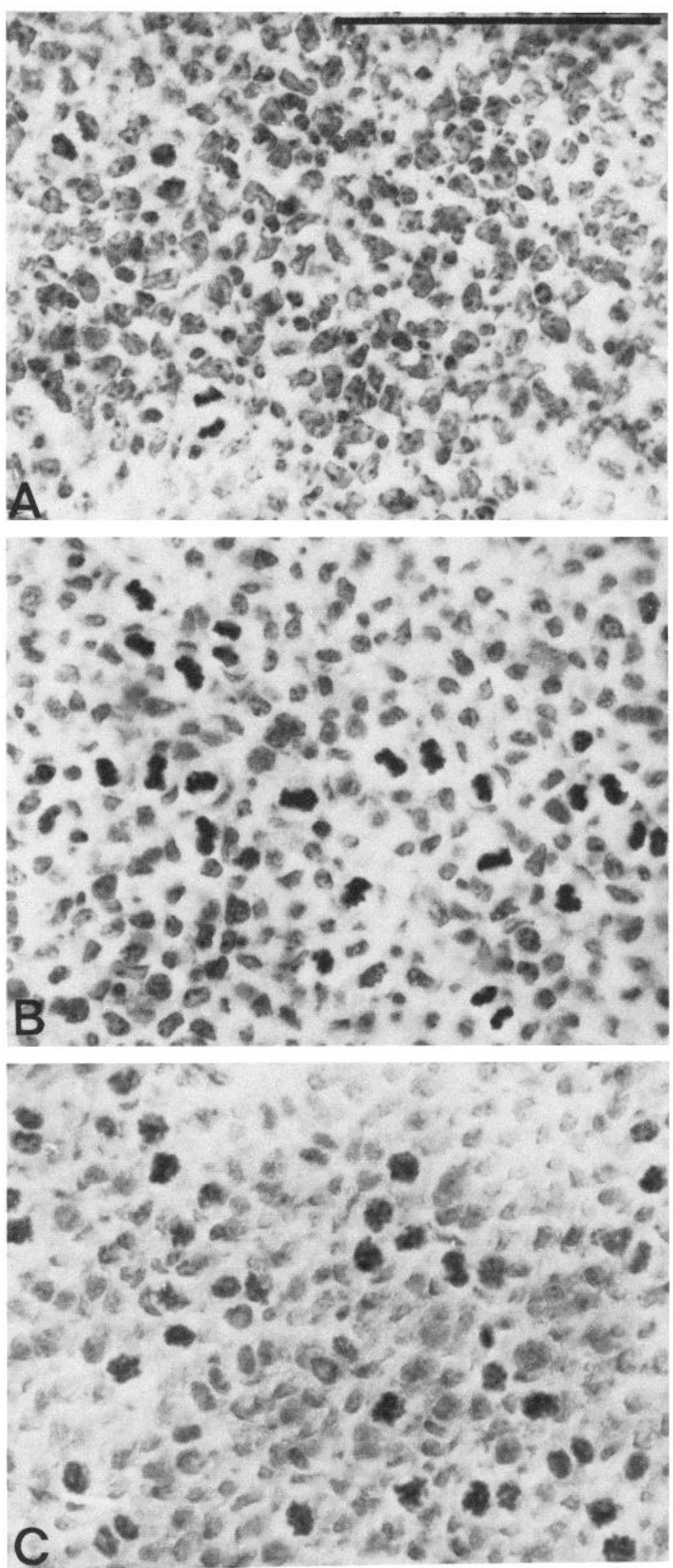

Figure 5. Regions of the ventricular layer of the retina of an E50 fetus. A, Region of near-zero mitotic activity at the site of the developing area centralis. One mitotic cell is present, demonstrating that the plane of focus is the ventricular layer. $B$, Mid-peripheral region showing the predominance of cells in metaphase, anaphase, or telophase. Note the almost complete absence of cells in prophase. $C$, Region near the edge of the retina, showing the common occurrence of cells in prophase. The scale bar represents $50 \mu$. 


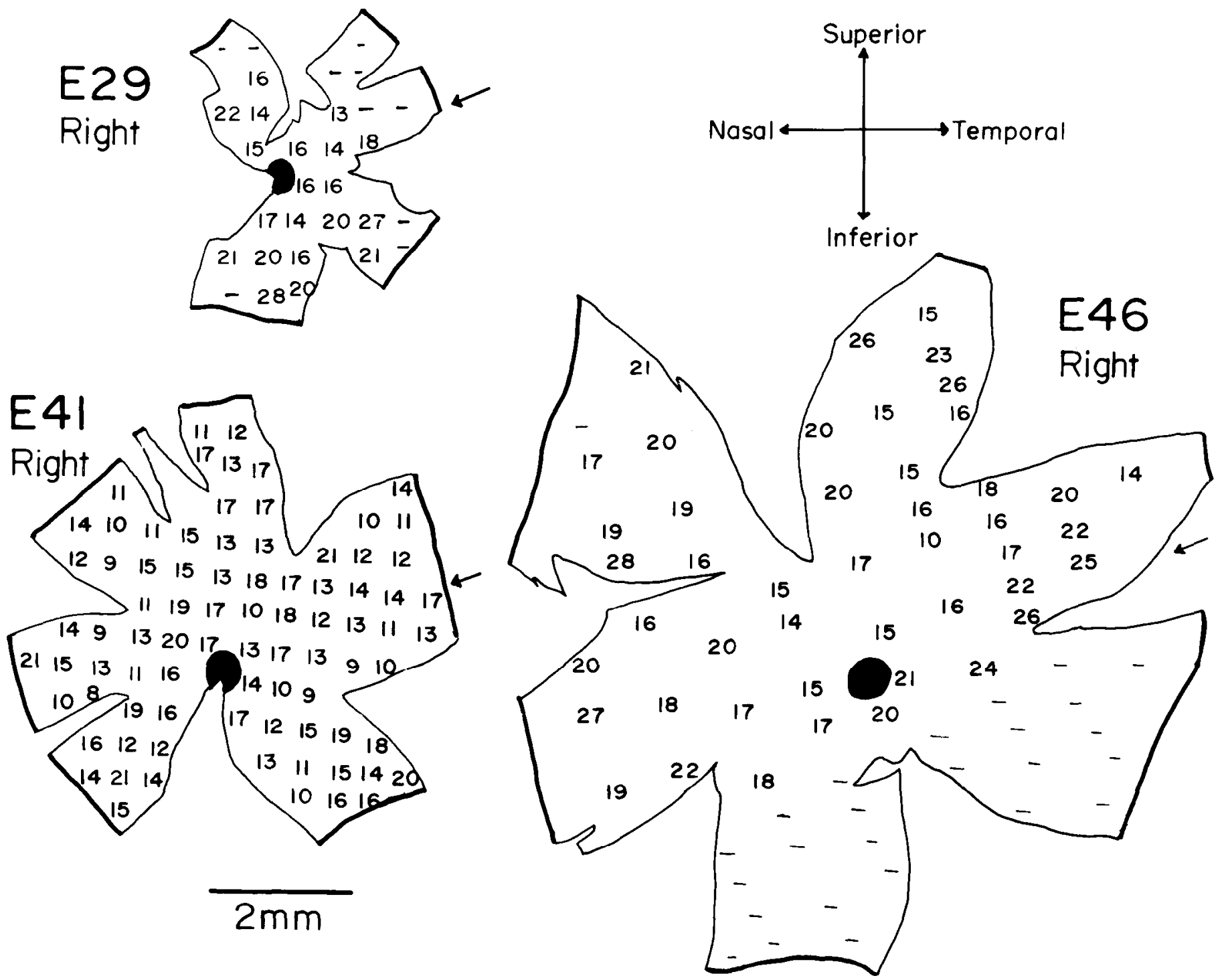

Figure 6. Maps of the distribution of mitotic cells in the retinae of three animals younger than E50. Each count represents the number of cells in an area of $0.01 \mathrm{~mm}^{2}$. No "cold spot" was apparent at any of these three ages; mitotic cells appeared to be distributed approximately uniformly. The dashes represent sites at which counts could not be made, usually because of adherent pigment cpithelium. The arrow on each map points to the optic disc across the region of the retina in which the area centralis will develop.

its edge. The distribution at E57 is of interest, for it shows most clearly the horizontal elongation of the cool region of reduced ( 200 to 2000 cells $/ \mathrm{mm}^{2}$ ) mitotic activity. Overall, the ratio of the horizontal of this region to its vertical extent is $1.4: 1$, but note the particular elongation near the nasal edge of the retina. For the cool regions at $\mathrm{P} 1$ and $\mathrm{P} 5$, the horizontal-to-vertical ratios were $1.1: 1$ and $1.2: 1$, respectively. Thus the ratio reduces as the region expands to include the whole retina, for the whole retina is not itself significantly elongated horizontally.

\section{Regional variations in the proportion of different stages of mitosis}

During the systematic counting required for the maps in Figures 5 and 7 we noted that the proportions of cells in the different stages of mitosis varied systematically with position in the retina. Specifically, the proportion of mitotic cells in prophase was relatively high throughout retinae from animals younger than E50. For example, in the E29 and E41 retinae prophase cells formed $52 \%$ and $66 \%$ (respectively) of the population of mitotic cells, while metaphase cells formed $15 \%$ and $4 \%$, and anaphase and telophase cells formed $33 \%$ and $30 \%$. These proportions seemed independent of position in the retina.

By E50, marked nonuniformities had appeared in this early pattern. The high proportion of prophase cells characteristic of younger retinae is found only in the peripheral $44 \%$ of the retina (Fig. 9, top). More centrally, cells in prophase form only 20 to $30 \%$ of the population of mitotic cells, and cells in metaphase are correspondingly more numerous (approximately $50 \%$ of mitotic cells). This change in proportions is quite obvious to inspection (compare Fig. 5, $B$ and $C$ ). The proportion of 

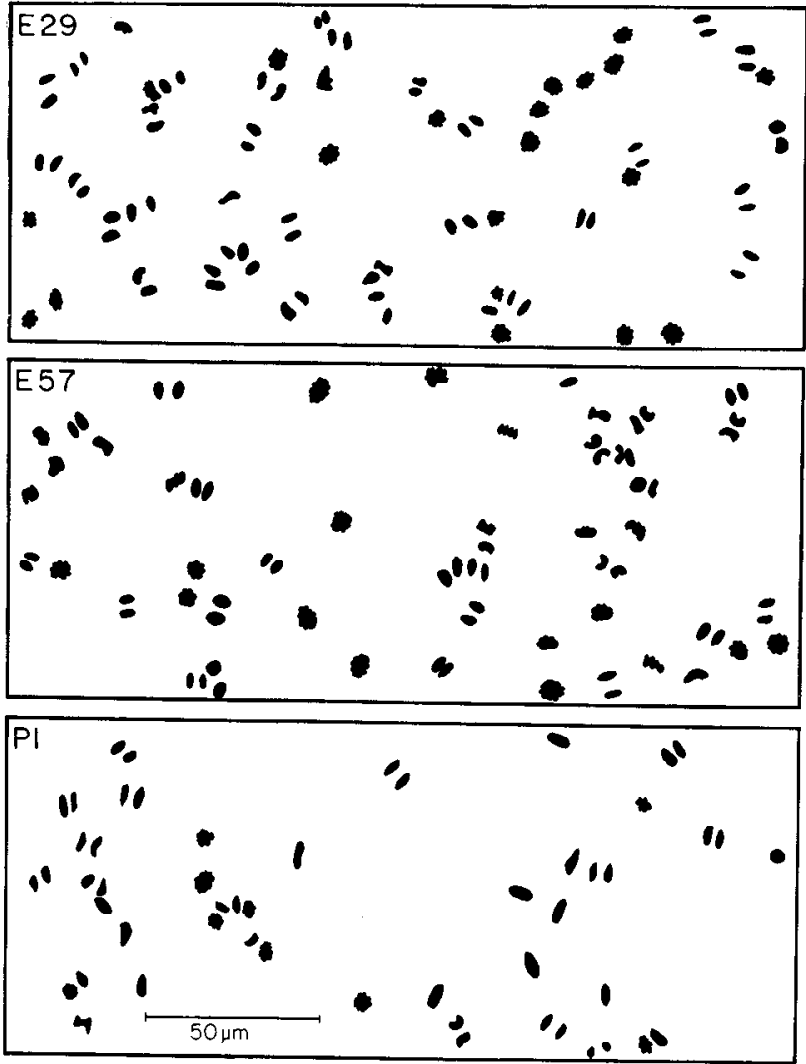

Figure 7. Drawings of the chromosomal material in mitotic cells in fetuses of increasing age. The sizes and shapes were remarkably constant, despite the great increase in the size and maturation of the retina during this period.

cells in anaphase or telophase is also higher in central than in peripheral retina, but by only a small margin; the major changes in proportions involve cells in prophase and metaphase. Figure 9, top, is a map of an E50 retina (the same as in Fig. 5) in which the region in which prophase cells comprise more than $50 \%$ of the mitotic population is represented diagrammatically. The map is schematic in that it represents the border between areas with high and low proportions of prophase cells as a sharp line. The graph in Figure 9 (bollom) shows, however, that the transition between these two areas is indeed quite sharp, at least at this age. Note also that the transition lies well outside the boundaries of the region of reduced mitotic activity.

At E57, an analysis similar to that in Figure 9 (bottom) showed that cells in prophase were found in high proportions $(>40 \%)$ only in the most peripheral regions of the retina, 1 to $2 \mathrm{~mm}$ from the edge. More centrally, cells in metaphase were relatively more common (up to $50 \%$ of the population of mitotic cells). Cells in anaphase and telophase comprised a relatively constant 30 to $40 \%$ of the population of mitotic cells. By P1, when mitotic cells are found only in the peripheral $50 \%$ of the retina, metaphase cells were relatively frequent $(48 \%)$, and prophase cells were infrequent $(13 \%)$ in all mitotic areas. Anaphase and telophase cells formed the remaining $39 \%$ of mitotic cells.

In summary, it seems that in regions of the retina in
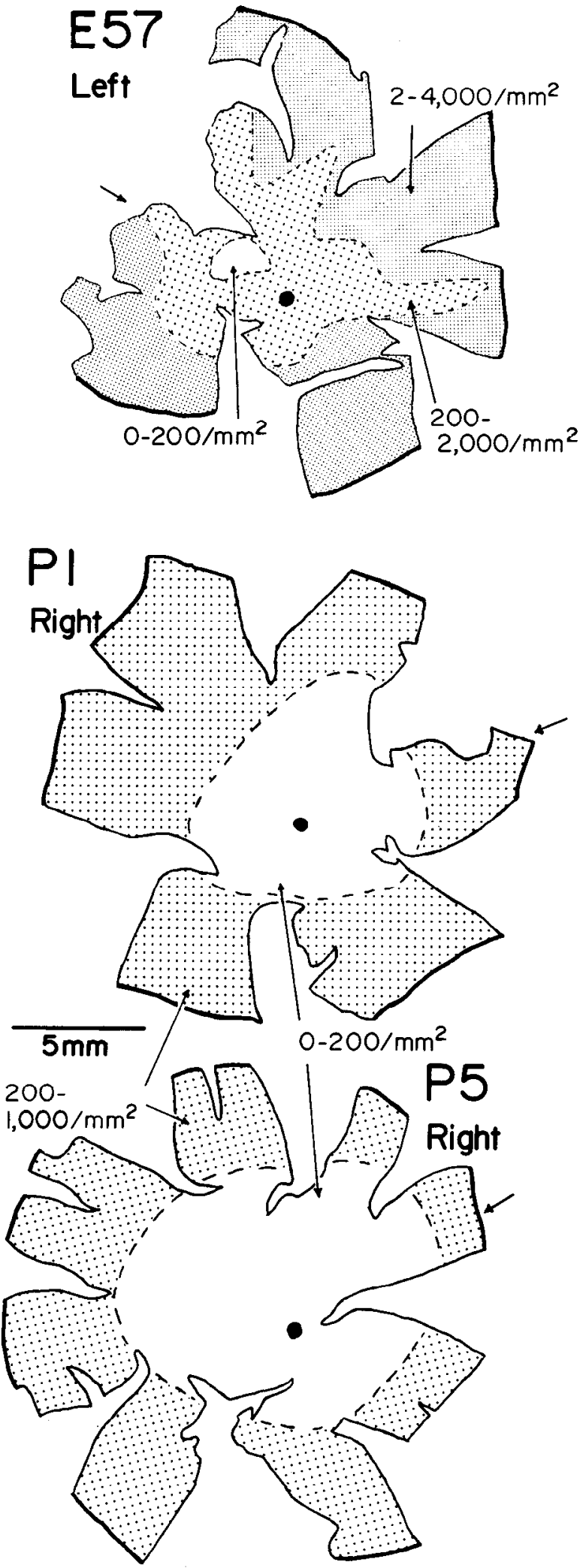

Figure 8. Maps of the distribution of mitotic cells in the retinae of three animals older than $\mathrm{E} 50$. The cold spot of nearzero $\left(<200\right.$ mitotic cells $/ \mathrm{mm}^{2}$ ) activity and the surrounding region of relatively low activity (200 to 1000 cells $/ \mathrm{mm}^{2}$, shown with coarse dots) expand steadily. By P10, no mitotic activity was apparent in the ventricular layer. In each map the arrow points to the optic disc across the area centralis. 

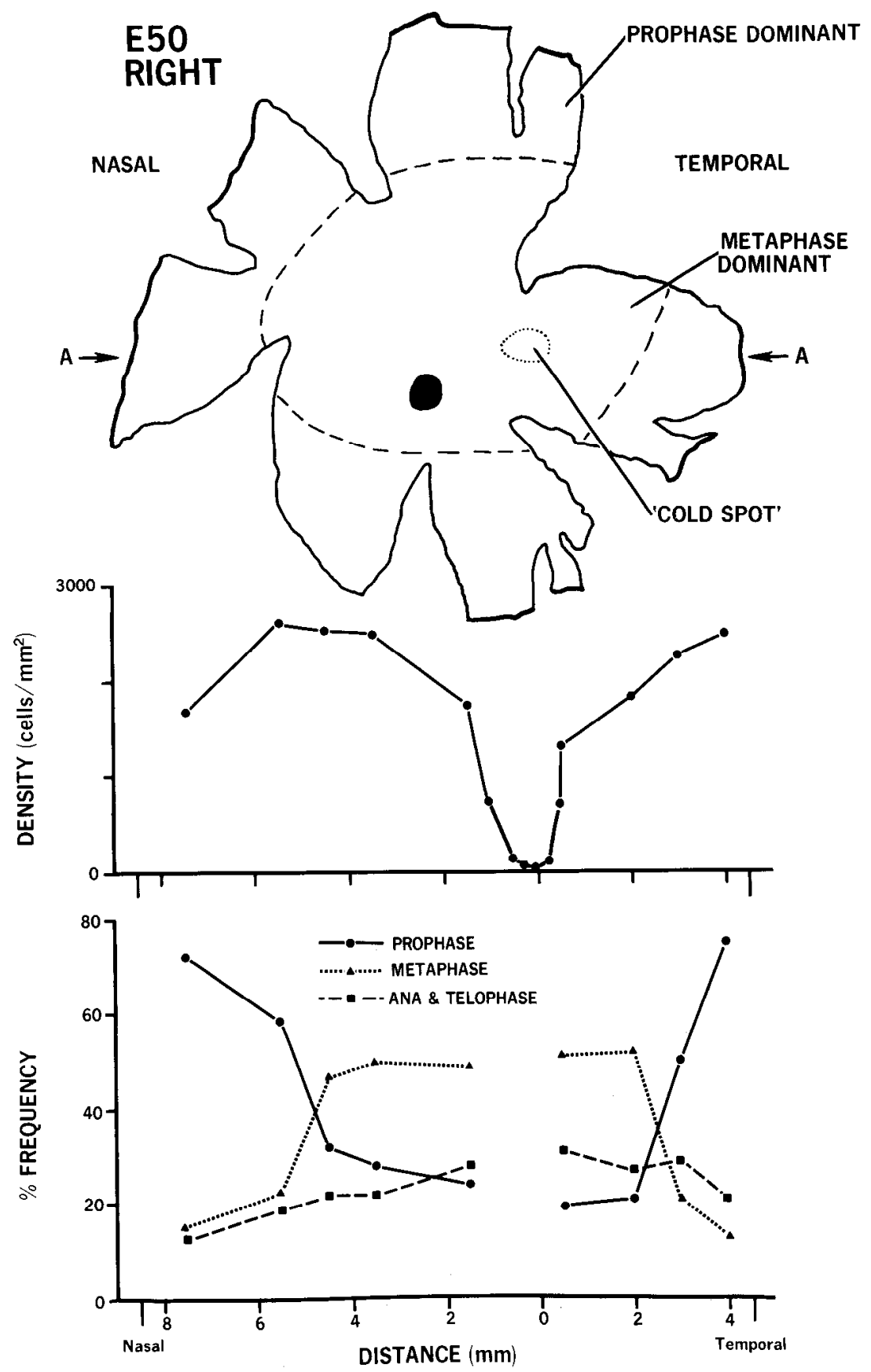

Figure 9. Analysis of the spatial distribution of prophase- and metaphasedominant mitotic activity in an E50 retina (same as in Fig. 5). Top, Map summarizing the pattern observed. Cells in prophase dominate in the peripheral regions of the retina, cells in metaphase dominate in the central regions. The arrows marked $A$ indicate the transect along which counts were made for the graphs below. Middle, Density of mitotic cells along the horizontal transect A-A in the map at the top. The transect passes through the area centralis, and the fall in mitotic cell density at the area centralis is obvious. Bottom, Proportions of cells in prophase, metaphase, and ana- plus telophase along the same transect. The transition from prophase dominance to metaphase dominance is quite sharp, and lies peripheral to the central region of low mitotic density. There is relatively little change in the proportion of anaphase and telophase cells. 
which mitotic activity will continue for a week or more, cells in prophase predominate over cells in other stages of mitosis. About a week before there is a reduction in the spatial density of mitotic cells, the proportion of cells in prophase decreases sharply and the proportion in metaphase increases correspondingly; the proportion in anaphase and telophase remains approximately constant. These new proportions are maintained for about a week, until cell division ceases.

\section{Discussion}

\section{The topography of cytogenesis}

We have studied the topography of mitotic cells in the ventricular layer of the developing retina of the cat, from E29 to P10. During this period:

1. The size and morphology of mitotic cells appear constant.

2. Until cytogenesis begins to cease, between E46 and E50, the density of mitotic cells ranges from approximately 1000 to 3000 cells $/ \mathrm{mm}^{2}$, with no consistent variation related to position.

3 . The cessation of cytogenesis occurs in a clear spatial pattern. At E50, a small region of zero mitotic activity (a "cold spot") is apparent at the site of the developing area centralis, surrounded by a narrow "cool" zone of relatively low mitotic activity. By birth, the cold spot has expanded to include the central 30 to $50 \%$ of the retina, and the level of mitotic activity in peripheral regions is relatively low $\left(<1000\right.$ cells $\left./ \mathrm{mm}^{2}\right)$. Mitotic activity in the ventricular layer ceases altogether between P5 and P10.

4. As they enlarge, the cold spot and cool zone are not circular in outline, but horizontally elongated, in a pattern resembling the visual streak specialization of the adult retina.

5 . We noted a systematic variation in the proportions of cells in different stages of mitosis. Cells in prophase predominate in regions of the retina in relatively early stages of cytogenesis. About a week before there is any decline in the spatial density of mitotic cells in a particular area of retina, the proportion of cells in prophase falls sharply, and the proportion in metaphase rises correspondingly.

Relationship to previous work. These results confirm many of the observations of Donovan (1966), Johns et al. (1979), and Greiner and Weidman (1980) on cytogenesis in the cat's retina. Both Donovan (1966) and Johns et al. (1979) described, for example, the relatively mature structure of the central regions of the retina at birth and the absence of mitotic activity there, and they noted the progressive spread of maturation over more peripheral regions of retina; also, Greiner and Weidman (1980) noted evidence of a decrease in the occurrence of mitotic cells at around E50. However, there are two quantitative discrepancies between our results and those of Johns et al. (1979). One discrepancy concerns the proportion of the retina over which cytogenesis has ceased at birth: Johns et al. (1979) estimated that proportion at $20 \%$, whereas our estimate is 30 to $50 \%$. This discrepancy may be more apparent than real, for Johns et al. (1979) observed the region of the retina of a 14-week-old kitten in which no cells were labeled by tritiated thymidine injected at $\mathrm{P} 1$, whereas we observed the nonmitotic area of a P1 retina. If, as Mastronarde et al. (1980) suggest, the peripheral regions of retina expand more during postnatal growth than do central regions, then the nonmitotic area of a P1 retina might comprise $40 \%$ of the retina at that age, but a considerably smaller proportion of an older retina. The other discrepancy seems less easily resolved. We observed mitotic cells only up to P10, whereas Johns et al. (1979) labeled some cells with tritiated thymidine injected as late as P21. Either the thymidine technique is more sensitive than observation of mitotic cells in Nissl-stained material, or some mitoses are taking place in inner layers of the retina, as in the goldfish (Johns, 1982), where they would not be seen in whole mount preparations.

Some support for this latter idea comes from the following considerations. In the telencephalon of the monkey (Schmechel and Rakic, 1979) the last cells to undergo mitosis are neuroglial cells, and their mitoses occur in the subventricular zone, some distance from the ventricular layer. A similar pattern may be followed in the retina. For example, Sidman (1961) noted that in the mouse retina the neuroglial cells of the ganglion cell layer are among the last to divide, and Johns et al. (1979) noted in the cat that the last-born cells have their somas in the inner nuclear layer, where the somas of Müller cells are found. However, neither author noted evidence of mitosis occurring away from the ventricular surface, and the idea requires testing.

\section{The obliquity of spindle axes}

The "interkinetic migration" model of cytogenesis in the vertebrate central nervous system was proposed by Sauer (1935). The nuclei of cells undergoing mitosis are located close to the ventricular margin, and the cells are believed to be attached to the ependymal lining of the ventricle. Sauer's model envisages that, in each mitosis, the mitotic spindle forms with its long axis parallel to the ventricular surface. Both daughter cells then form at the ventricular surface and remain attached to it. However, the daughter cells elongate, allowing their nuclei to move away from the ventricular surface. Prior to its next mitosis the cell contracts to the ventricular margin. Many studies, including that of Martin (1967), have confirmed Sauer's (1935) observation that spindles of most mitotic cells in the ventricular layer are parallel to the ventricular surface. Our results confirm this pattern, for the retina. Martin (1967) drew renewed attention to the minority of mitotic spindles which are oriented obliquely to the ventricular surface. He suggested that, during a "final" mitosis, the spindle is obliquely oriented, so that one daughter cell is formed a short distance from the ventricular surface. Therefore, it does not attach to that surface and is free to migrate to its final position.

Although a number of highly oblique spindles were observed at all of the ages examined (Fig. 3), neither the mean obliquity of spindles nor the proportion of highly oblique spindles changed significantly between E32 and P1, i.e., between mid- and late stages of cytogenesis. In terms of Martin's suggestion, the proportion of final mitoses (among all mitoses) does not change markedly during cytogenesis. 


\section{Do the phases of the mitotic cycle vary in duration?}

We have noted that about a week before the spatial density of mitotic cells in the retina begins to decline there is a shift in the proportions of cells in different stages of the mitotic cycle. Cells in prophase are the most common form in regions of the retina in relatively early stages of their cytogenic life; for example, in the E29 and $\mathrm{E} 41$ retinae, and in the peripheral margins of the E50 and $\mathrm{E} 57$ retinae. In regions of the retina nearer the end of cytogenesis (such as the central regions of the E50 and E57 retinae, and the margins of the P1 and P5 retinae) cells in prophase are much less common and cells in metaphase are correspondingly more frequent. Cells in anaphase and telophase form a relatively constant proportion of mitotic cells during cytogenesis. The simplest explanation of this shift is that the relative times which a mitotic cell spends in prophase and metaphase change systematically. Since the mitotic cycle generally lengthens with increasing age, it seems reasonable to suggest that at late stages of cytogenesis the time spent by cells in metaphase increases, so that this phase is seen more frequently. Since the proportions of cells in anaphase and telophase do not drop, the times spent in these phases presumably also increase, although to a lesser extent. Perhaps there is a slowing down toward the end of cytogenesis in the kinetics of the formation of the microtubules which comprise mitotic spindles.

\section{Mechanisms of maturation}

In the context of our own recent work, the most striking feature of the present results is the near-simultaneity of major developmental events in the several laminae of the retina. The cessation of cytogenesis begins at the same place (the region of the area centralis) and at a similar time (E46 to E50) as the formation of the outer plexiform layer (OPL) (Rapaport and Stone, 1982), and proceeds with a similar time course and pattern. Thus, the OPL spreads toward the edge of the retina, and cytogenesis is increasingly restricted to the edge of the retina. Both processes are complete by P5 to P10, and in intermediate stages of both, the pattern of spread is horizontally elongated. The formation of the OPL presumably is a measure of the formation of synaptic connections between cells of the outer and inner nuclear layers. It would seem, therefore, that synaptogenesis does not begin until cytogenesis has stopped; but it then proceeds promptly.

Furthermore, the cessation of cytogenesis begins at approximately the same time as a process of maturation in the ganglion cell layer (Stone et al., 1982). None of our results indicate the causal mechanisms in these processes, but it seems clear that the maturation of the retina which begins at E46 to E50 affects all of its layers, with closely matched spatial and temporal patterns. It may therefore be determined by a single, intraretinal mechanism. Perhaps, for example, the strictly radial direction of migration of cells from the ventricular layer to their final position enables regional variation in the ventricular layer to determine regional variation in the inner layers of the retina. Rakic (1981) has envisaged, for example, that the ventricular layer of the telencephalon might determine regional variations in the cere- bral cortex, and Cooper and Rakic (1981) have suggested a similar determinant for regional variations in the roof of the mesencephalon.

This idea of an intraretinal determination of the maturational processes of the retina (discussed also in $\mathrm{Ra}$ paport and Stone, 1982, 1983) is an alternative to the suggestion made in several studies (Rager and Rager, 1976; Hinds and Hinds, 1978; Hughes and McLoon, 1979; Stone et al., 1982) that the maturation of the retina and, in particular, of the ganglion cell layer, is determined by a competition among the axons of ganglion cells for synaptic space in the visual centers of the brain. It should be noted, however, that the two ideas are not mutually exclusive.

\section{Determinants of adult topography}

Two features of the present results seem of significance for an understanding of how the regional specialization of the adult retina arises. First, cytogenesis ceases initially in the region of the area centralis; second, the visual streak specialization is reflected in the pattern of cessation. It seems paradoxical, however, that cytogenesis ceases first in regions of the retina which are characterized in the adult by a high concentration of ganglion cells. This lends support to earlier suggestions by ourselves (Stone et al., 1982) and others (Kliot and Shatz, 1982) that the concentrations of ganglion cells prominent in the adult retina are not directly produced by patterns in the timing or location of the genesis of ganglion cells.

Alternative mechanisms for the formation of the regional specializations of the retina need to be considered, therefore, such as differential cell death or maturation, and differential retinal growth (reviewed in Stone et al., 1982). It is difficult to add to these ideas from the present results, because of one intrinsic limitation to them: we do not know the destiny of the daughter cells of the mitoses we observed. The work of Polley et al. (Polley et al., 1981; Walsh et al., 1983) and Kliot and Shatz (1982) makes clear, for example, that all ganglion cells in the cat's retina are born by E35. The pattern of cessation of cytogenesis which is discernible after E50 is thus directly relevant only to cells of the outer layers of the retina.

One positive suggestion of the developmental basis of adult topography does seem available from the present results: it is a remarkable feature of the adult retina that the number of cells in the outer nuclear layer (and, correspondingly, the density of receptors (Steinberg et al., 1973)) are approximately constant between the area centralis and the edge of the retina, despite the concurrent attenuation of the ganglion cell population. The continuation of cytogenesis in the peripheral margins of the retina into early postnatal life may be the source of these relatively high numbers of receptor cells in the peripheral regions of the adult retina. Thus the high number of receptors in peripheral retina may be produced by patterns in the timing and location of their genesis.

\section{References}

Angevine, J. B., D. Bodian, A. J. Coulombre, M. V. Edds, V. Hamburger, M. Jacobsen, K. M. Lyser, M. C. Prestige, R. L. Sidman, S. Varon, and P. A. Weiss (The Boulder Committee) (1970) Embryonic vertebrate central nervous system: Revised terminology. Anat Rec. 166: 257-262. 
Cooper, M. L., and P. Rakic (1981) Neurogenetic gradients in the superior and inferior colliculi of the rhesus monkey. J. Comp. Neurol. 202: 309-334.

Donovan, A. (1966) The postnatal development of the cat's retina. Exp. Eye Res. 5: 249-254.

Greiner, J. V., and T. A. Weidman (1980) Histogenesis of the cat retina. Exp. Eye Res. 30: 439-453.

Hinds, J. W., and P. L. Hinds (1978) Development of amacrine cells in the mouse retina: An electron microscopic, serial section analysis. J. Comp. Neurol. 179: 277-300.

Hughes, W. F., and S. C. McLoon (1979) Ganglion cell death during normal retinal development in the chick: Comparison with cell death induced by early target field destruction. Exp. Neurol. 66: 587-601.

Johns, P. R. (1982) Formation of photoreceptors in larval and adult goldfish, J. Neurosci. 2: 178-198.

Johns, P. R., A. C. Rusoff, and M. W. Dubin (1979) Postnatal neurogenesis in the kitten retina. J. Comp. Neurol. 187: 545556.

Kliot, M., and C. J. Shatz (1982) Genesis of different retinal ganglion cell types in the cat. Soc. Neurosci. Abstr. 8: 815.

Martin, A. H. (1967) Significance of mitotic spindle orientation in the neural tube. Nature 216: 1133-1134.

Mastronarde, D. N., M. A. Thibeault, and M. W. Dubin (1980) How ganglion cells redistribute during postnatal growth of the cat's retina. Invest. Opthalmol. (Suppl.) 19: 70.

Morest, D. K. (1970) The pattern of neurogenesis in the retina of the rat. Z. Anat. Entwicklungsgesch. 131: 45-67.

$\mathrm{Ng}, \mathrm{A}$. Y. K., and J. Stone (1982) The optic nerve of the cat: Appearance and loss of axons during normal development. Dev. Brain Res. 5: 263-271.

Polley, E. H., C. Walsh, and T. L. Hickey (1981) Neurogenesis in cat retina. A study using ${ }^{3} \mathrm{H}$-thymidine autoradiography.
Soc. Neurosci. Abstr. 7: 672.

Rager, G., and U. Rager (1976) Generation and degeneration of retinal ganglion cells in the chicken. Exp. Brain Res. 25: $551-553$.

Rakic, P. (1981) Neuronal-glial interaction during brain development. Trends Neurosci. 4: 184-187.

Rapaport, D. H., and J. Stone (1982) The site of commencement of maturation in mammalian retina: Observations in the cat. Dev. Brain Res. 5: 273-279.

Rapaport, D. H., and J. Stone (1983) The area centralis in the cat and other mammals: Focal point for function and development of the visual system. Neuroscience, in press.

Sauer, F. C. (1935) Mitosis in the neural tube. J. Comp. Neurol. 62: $377-405$.

Schmechel, D. E., and P. Rakic (1979) Arrested proliferation of radial glial cells during midgestation in rhesus monkey. Nature 277: 303-305.

Sidman, R. L. (1961) Histogenesis of mouse retina studied with thymidine- $\mathrm{H}^{3}$. In The Structure of the Eye, G. Smelser, ed., pp. 487-505, Academic Press, Inc., New York.

Steinberg, R. H., M. Reid, and P. L. Lacy (1973) The distribution of rods and cones in the retina of the cat (Felis domesticus). J. Comp. Neurol. 148: 229-248.

Stone, J. (1981) The Wholemount Handbook, Maitland, Sydney, Australia.

Stone, J., D. H. Rapaport, R. W. Williams, and L. Chalupa (1982) Uniformity of cell distribution in the ganglion cell layer of prenatal cat retina: Implications for mechanisms of retinal development. Dev. Brain Res. 2: 231-242.

Walsh, C., E. H. Polley, T. L. Hickey, and R. W. Guillery (1983) Generation of cat retinal ganglion cells in relation to central pathways. Nature 302: 611-614. 